\title{
NMR Determination of Smectic Ordering of Probe Molecules
}

\author{
Anand Yethiraj, ${ }^{\dagger, \ddagger}$ Adrian C. J. Weber, ${ }^{\dagger}$ Ronald Y. Dong, ${ }^{\S}$ and E. Elliott Burnell ${ }^{*}, \dagger$ \\ Chemistry Department, University of British Columbia, 2036 Main Mall, Vancouver, BC, V6T 1Z1, Canada, \\ and Department of Physics and Astronomy, Brandon University, Brandon, MB, R7A 6A9, Canada
}

Received: October 27, 2006; In Final Form: December 6, 2006

\begin{abstract}
The NMR spectra of the three solutes ortho-, meta-, and para-dichlorobenzene in the nematic and smectic A phases of the liquid crystals $8 \mathrm{CB}$ and $80 \mathrm{CB}$ are analyzed to yield two orientational order parameters for each solute. Extrapolation of the asymmetry in the energy parameters that describe the orientational ordering in the nematic phase are used to provide estimates of the strength of the nematic potential in the smectic A phase. The experimentally determined asymmetry of the orientational order parameters in the smectic A phase is then used in conjunction with Kobayashi-McMillan theory applied to solutes to give information about the smectic A layering and the nematic/smectic A coupling. In both smectic A solvents, the solute smectic coupling constant, $\tau$, is negative (with the origin fixed at the center of the smectic layer) for all solutes. The signs and relative values of $\tau$ indicate that the ortho and para solutes favor the interlayer region while the meta solute is more evenly distributed throughout the layers.
\end{abstract}

\section{Introduction}

An understanding of the physical properties of liquid crystalline systems is key to a host of problems spanning fields ranging from biological membranes to liquid crystal displays and other applications. ${ }^{1,2}$ An important property of such systems is the degree of orientational and positional order which is characterized by order parameters. Nematic liquid crystals have uniaxial orientational order along an average direction called the director. The molecules that make up most nematic phases are rodlike and normally have a rigid core with flexible hydrocarbon ends and have little symmetry; nevertheless, they are often approximated as axially symmetric rods and their NMR spectra are then determined by a single nematic order parameter, $S_{\mathrm{lc}}$. A more rigorous description would take into account the asymmetry of the molecules and their flexibility, and additional order parameters would be required. ${ }^{3}$ It is generally found that these additional parameters are quite small and the description of the nematic molecules as symmetric rods is sufficient to explain most of their properties. For the case of perfect order, that is, all rods aligned perfectly along the director, $S=1$. For an isotropic liquid in which the rods have random orientations, $S$ $=0$.

Smectic liquid crystals have positional order in addition to orientational order and in the simplest smectic A case form layers in which on average the molecules align along the normal to the layers which is also the director. Kobayashi-McMillan theory accounts for the positional order by introducing extra order parameters. ${ }^{4,5}$ The extent of layering is described by the smectic order parameter, $\tau$. However, smectic and nematic order parameters cannot be independent because $S_{\mathrm{lc}}$ is defined in the smectic phase, and increases as one goes deeper into the smectic phase. This coupling between increasing $S_{\mathrm{lc}}$ and more welldeveloped smectic order is expressed in Kobayashi-Mcmillan theory by the coupling order parameter, $\kappa$. While this mean-

\footnotetext{
$\dagger$ University of British Columbia.

Present address: Department of Physics and Physical Oceanography, Memorial University of Newfoundland, St. John's, NL, A1B3X7, Canada.

$\S$ Brandon University.
}

field theory is 30 years old, no understanding at the mean-field level is complete without experimental determination of prefactors, something that has not been undertaken until recently. ${ }^{6}$ Moreover, other curious observations have been made. While it is well-established that smectic order is well-described by a sinusoidal modulation, ${ }^{7}$ recent observations using singlemolecule imaging ${ }^{8}$ of long-chain molecules dissolved in the smectic A phase find that $10 \%$ of the molecules lie perpendicular to the nematic director, an observation that would make better sense if the smectic ordering were truly perfect (square-wave) layering. Thus, the understanding of the details of how molecules might position themselves within the layers has much currency.

NMR is a powerful technique for measuring orientational order parameters. Often the liquid crystal molecules have low symmetry and contain flexible end chain(s), making the determination of orientational order parameters difficult. Small solutes have been successfully used as probes of the anisotropic intermolecular potential that causes orientational order in nematic ${ }^{9-11}$ liquid crystals. While orientational order in smectic $^{6,12-16}$ and columnar ${ }^{17-19}$ liquid crystals has been probed, the determination of positional order parameters is not as straightforward and there is no NMR measurement that relates directly to these quantities in Kobayashi-McMillan theory.

Recently, we have explored the use of solutes for the investigation of both orientational and positional order in a smectic A liquid crystal. ${ }^{6}$ In a preliminary communication, the NMR spectra of para-dichlorobenzene and fluorobenzene (in each case, the second-rank orientational order is described by two independent orientational order parameters) dissolved in the liquid crystal $8 \mathrm{CB}$ were interpreted in terms of the smectic A ordering and coupling potentials experienced by the solutes in the smectic A phase of this liquid crystal. ${ }^{6}$ KobayashiMcMillan theory ${ }^{4,5}$ for smectic A liquid crystals was modified to describe the ordering of solutes in such phases. The theory adds smectic order and coupling terms to the original MaierSaupe theory ${ }^{20,21}$ that has been so successful for the description of nematics. The derived interaction parameters (in particular the coupling between the nematic ordering and the layers) in 


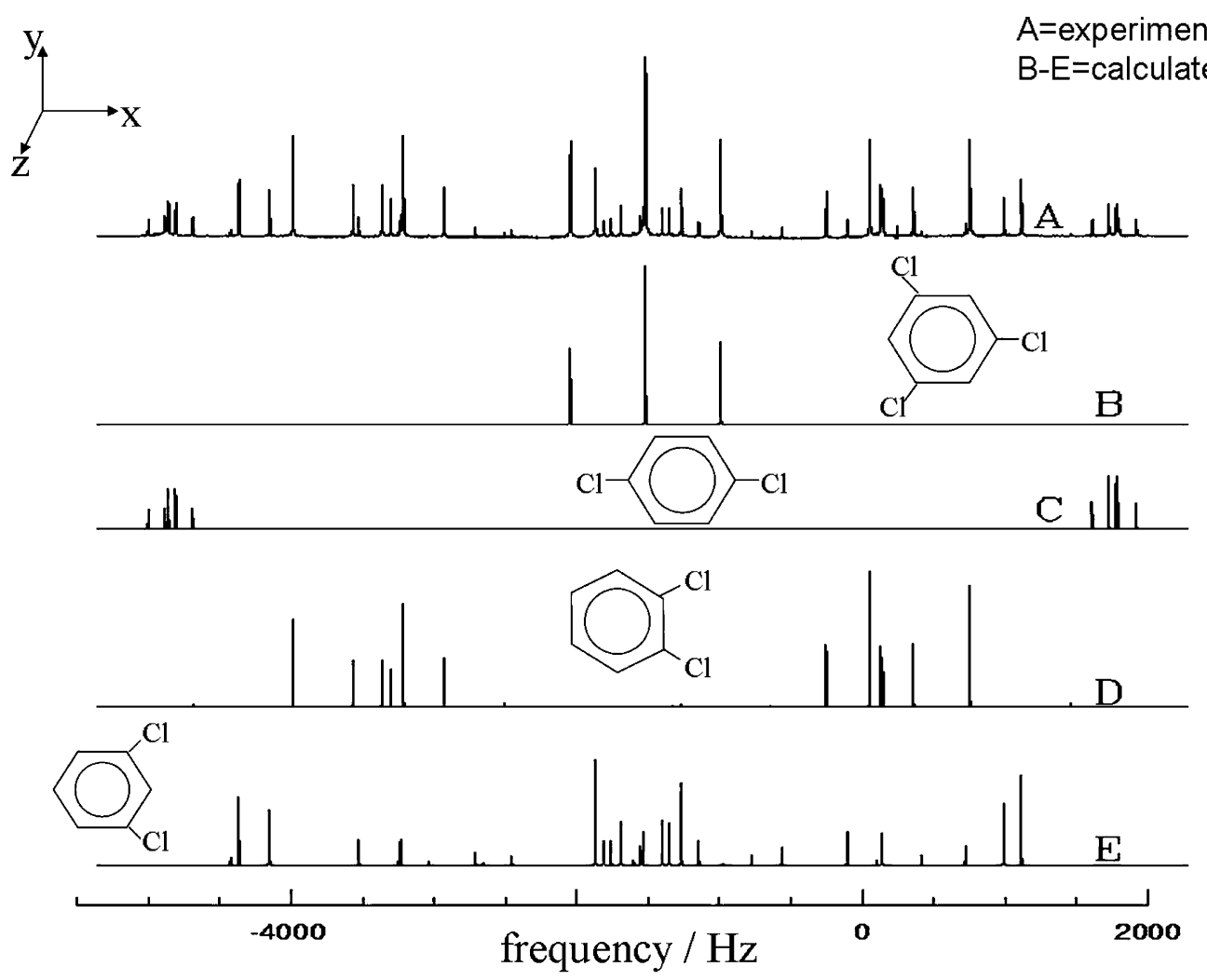

Figure 1. Experimental (top) and calculated spectra of the solutes tcb, pdcb, odcb, and mdcb in $8 \mathrm{CB}$ at $295.5 \mathrm{~K}$. The frequency-scale origin is arbitrary.

our approach seemed to contrast with our expectation. In other words, the coupling strength, $\kappa$, between the orientational potential and the smectic term was found to be unrealistically strong. As a consequence, we decided to test further the ideas put forward in the earlier paper by investigating smectic A phases with NMR experiments on additional solutes in two liquid crystals that have a nematic phase and a lower-temperature smectic A phase. Moreover, it is expected that the strengths of both smectic and coupling terms depend on temperature. In the present work, a new temperature scaling of the smectic potential is proposed. This improvement has led to better results that support the application of Kobayashi-McMillan mean-field theory of smectic A phases based on NMR.

\section{Experimental Section}

About $1 \mathrm{~mol} \%$ of each of the solutes ortho-dichlorobenzene (odcb), meta-dichlorobenzene (mdcb), para-dichlorobenzene (pdcb), and 1,3,5-trichlorobenzene (tcb) were codissolved in the liquid crystals 4- $n$-octyl-4'-cyanobiphenyl (8CB) and 4- $n$ octyloxy-4'-cyanobiphenyl (8OCB). The tcb was added as an orientational reference but was not used in the work reported here. The samples were well mixed in the isotropic phase, and all experiments started with the sample in the nematic phase in which the director is oriented by the magnetic field along the field direction. Spectra were acquired on a Bruker Avance 400 MHz NMR spectrometer with the temperature controlled by the Bruker air-flow system. Spectra were acquired throughout the nematic and smectic A phases, usually in 0.5 or $1{ }^{\circ} \mathrm{C}$ steps.

The four superimposed spectra in each sample at each temperature were analyzed with the computer program LEQUOR $^{22}$ using $J$ couplings from the literature 23,24 to obtain the NMR dipolar couplings and chemical shifts; an example of an experimental spectrum along with the fitted spectra of the solutes is shown in Figure 1.
The dipolar couplings were analyzed using a modified version of the computer program $\mathrm{SHAPE}^{25}$ along with fixed structures from refs 23 and 24 to obtain the order parameters reported in Table 1 for each solute in each experiment.

Figure 2 shows the variation of $-S_{z z}$ with temperature. For both liquid crystals $8 \mathrm{CB}$ and $8 \mathrm{OCB}$, the solute order parameters hardly change at the nematic/smectic A phase transition. However, as found in the earlier study, ${ }^{6}$ the transition for each solute is marked by a change in the slope of the solute order matrix asymmetry

$$
R=\left(S_{x x}-S_{y y}\right) / S_{z z}
$$

(plotted versus parameters such as temperature, solute $S_{x x}$, etc.), as shown in Figure 3. It is this change that is key to extracting information about the smectic potential. Note that the definition of $R$ in this paper is minus the quantity used in ref 6 .

\section{Theoretical Considerations}

Maier-Saupe mean-field theory for the orientational ordering of nematic liquid crystals ${ }^{20,21}$ starts from a pairwise interaction between two axially symmetric rodlike particles and leads to a description in which each particle can be considered to be interacting with the mean field of the other particles by the second-rank interaction:

$$
H_{\mathrm{N}}^{\mathrm{lc}}=-v_{\mathrm{lc}} S_{1 \mathrm{c}} P_{2}(\cos \theta)
$$

where $v_{\mathrm{lc}}$ is a scale parameter that indicates the strength of the interparticle interaction and $S_{\mathrm{lc}}=\left\langle P_{2}(\cos \theta)\right\rangle=\left\langle(3 / 2) \cos ^{2}(\theta)\right.$ $-(1 / 2)\rangle$ is the nematic order parameter; $\theta$ is the angle between the long rod axis and the mean field, and the angle brackets denote an average. 
TABLE 1: Order Parameters Calculated from the Dipolar Couplings

\begin{tabular}{|c|c|c|c|c|c|c|}
\hline \multirow[b]{2}{*}{$T / \mathrm{K}$} & \multicolumn{2}{|c|}{$\mathrm{odcb}^{a}$} & \multicolumn{2}{|c|}{$\mathrm{mdcb}^{a}$} & \multicolumn{2}{|c|}{$\mathrm{pdcb}^{b}$} \\
\hline & $S_{x x}$ & $S_{z z}$ & $S_{x x}$ & $S_{z z}$ & $S_{x x}$ & $S_{z z}$ \\
\hline \multicolumn{7}{|c|}{$8 \mathrm{CB}$} \\
\hline 291.0 & 0.19987 & -0.24460 & 0.07593 & -0.24021 & 0.28912 & -0.24663 \\
\hline 292.0 & 0.19666 & -0.24218 & 0.07450 & -0.23817 & 0.28753 & -0.24515 \\
\hline 293.0 & 0.19349 & -0.23964 & 0.07268 & -0.23581 & 0.28412 & -0.24219 \\
\hline 293.5 & 0.19184 & -0.23831 & 0.07180 & -0.23462 & 0.28280 & -0.24102 \\
\hline 294.0 & 0.19019 & -0.23710 & 0.07090 & -0.23342 & 0.28150 & -0.23983 \\
\hline 294.5 & 0.18838 & -0.23557 & 0.06992 & -0.23207 & 0.27999 & -0.23857 \\
\hline 295.0 & 0.18658 & -0.23413 & 0.06901 & -0.23074 & 0.27851 & -0.23722 \\
\hline 295.5 & 0.18472 & -0.23262 & 0.06795 & -0.22929 & 0.27693 & -0.23583 \\
\hline 296.0 & 0.18280 & -0.23100 & 0.06716 & -0.22796 & 0.27529 & -0.23436 \\
\hline 296.5 & 0.18075 & -0.22932 & 0.06592 & -0.22626 & 0.27356 & -0.23282 \\
\hline 297.0 & 0.17874 & -0.22761 & 0.06495 & -0.22472 & 0.27174 & -0.23118 \\
\hline 297.5 & 0.17651 & -0.22568 & 0.06360 & -0.22282 & 0.26979 & -0.22954 \\
\hline 298.0 & 0.17413 & -0.22357 & 0.06251 & -0.22095 & 0.26758 & -0.22749 \\
\hline 298.5 & 0.16832 & -0.2 & 0.05949 & -0.2 & 0.26181 & -0.22236 \\
\hline 299.0 & 0.16455 & -0.21451 & 0.05754 & -0.21238 & 0.25807 & -0.21905 \\
\hline 299.5 & 0.15988 & -0.21000 & 0.05567 & -0.20845 & 0.25326 & -0.21473 \\
\hline 300.0 & 0.15293 & -0.20294 & 0.05192 & -0.20147 & 0.24570 & -0.20800 \\
\hline 300.5 & 0.14031 & -0.18917 & 0.04627 & -0.18835 & 0.23046 & -0.19464 \\
\hline 301.0 & 0.13419 & -0.18233 & 0.04379 & -0.18172 & 0.22246 & -0.18780 \\
\hline 301.5 & 0.12911 & -0.17642 & 0.04195 & -0.17604 & & -0.18205 \\
\hline 302.0 & 0.12456 & & 0.04026 & & & -0.17662 \\
\hline 302 & 0.11946 & & 0.03847 & -0.1 & 139 & -0.17035 \\
\hline 303.0 & 0.11396 & -0.15814 & 0.03682 & & 0.19311 & -0.16351 \\
\hline \multicolumn{7}{|c|}{$80 C B$} \\
\hline 316.3 & 0.18858 & -0.24057 & 0.06509 & -0.23683 & 0.29202 & -0.24469 \\
\hline 316.8 & 0.18742 & -0.23961 & 0.06459 & -0.23593 & 0.29090 & -0.24380 \\
\hline 317.3 & 0.18665 & -0.23897 & 0.06427 & -0.23 & 0.29016 & -0.24320 \\
\hline 317.8 & 0.18548 & -0.23798 & 0.06377 & -0.23441 & 0.28904 & -0.24229 \\
\hline 318.3 & 0.18440 & -0.23707 & 0.06330 & -0.23355 & 0.28800 & -0.24145 \\
\hline 318.8 & 0.18360 & -0.23639 & 0.06298 & -0.232 & 0.28723 & -0.24082 \\
\hline & 0.18237 & & 0.06245 & & 0.28603 & -0.23985 \\
\hline & 0.18127 & -0.2 & 0.06197 & -0.2 & 0.28496 & -0.23897 \\
\hline 20.3 & 0.18043 & -0.23368 & 0.06161 & -0.23039 & 0.28414 & -0.23830 \\
\hline 320.8 & 0.17917 & -0.23259 & 0.06105 & -0.22937 & 0.28291 & -0.23729 \\
\hline 321.3 & 0.17827 & -0.23180 & 0.06068 & -0.22865 & 0.28203 & -0.23655 \\
\hline 321.8 & 0.17714 & -0.23082 & 0.06017 & -0.22772 & 0.28091 & -0.23564 \\
\hline 322.3 & 0.17594 & -0.22976 & 0.05965 & -0.22674 & 0.27973 & -0.23466 \\
\hline 322.8 & 0.17509 & -0.22901 & 0.05928 & -0.22604 & 0.27888 & -0.23395 \\
\hline 323.3 & & & 0.05869 & & 0.27755 & -0.23285 \\
\hline 323.8 & 0.17273 & -0.22691 & 0.05822 & -0.22406 & 0.27652 & -0.23199 \\
\hline 324.3 & 0.17163 & -0.22592 & 0.05772 & -0.22314 & 0.27542 & -0.23106 \\
\hline 324.8 & 0.17040 & -0.22478 & 0.05716 & -0.22208 & 0.27417 & -0.23001 \\
\hline 325.3 & 0.16947 & -0.22396 & .05676 & -0.22131 & 0.27323 & -0.22922 \\
\hline 325.8 & 0.16806 & -0.22267 & 0.05614 & -0.22012 & 0.27180 & -0.22801 \\
\hline 326.3 & 0.16700 & -0.22168 & 0.05564 & -0.21918 & 0.27071 & -0.22709 \\
\hline 326.8 & 0.16571 & -0.22049 & 0.05505 & -0.21806 & 0.26939 & -0.22597 \\
\hline 327.3 & 0.16436 & & & & 0.26799 & -0.22477 \\
\hline 327.8 & 0.16339 & -0.2183 & 0.05399 & -0.216 & 0.26697 & -0.22390 \\
\hline 328.3 & 0.16179 & -0.21679 & 0.05326 & -0.21460 & 0.26530 & -0.22247 \\
\hline 328.8 & 0.16054 & -0.21559 & 0.05269 & -0.21347 & 0.26399 & -0.22134 \\
\hline 329.3 & 0.15918 & -0.21427 & 0.05207 & -0.21225 & 0.26254 & -0.22008 \\
\hline 329.8 & 0.15762 & -0.21276 & 0.05137 & -0.21083 & 0.26087 & -0.21864 \\
\hline 330.3 & 0.15635 & -0.21149 & 0.05073 & -0.20962 & 0.25949 & -0.21745 \\
\hline 330.8 & 0.15449 & -0.20965 & 0.04991 & -0.20789 & 0.25748 & -0.21570 \\
\hline 331.3 & 0.15287 & -0.20797 & 0.04914 & -0.20632 & 0.25565 & -0.21408 \\
\hline 331.8 & 0.15100 & -0.20609 & 0.04828 & -0.20455 & 0.25359 & -0.21228 \\
\hline 332.3 & 0.14903 & -0.20407 & 0.04736 & -0.20266 & 0.25142 & -0.21036 \\
\hline 332.8 & 0.14714 & -0.20209 & 0.04658 & -0.20086 & 0.24927 & -0.20849 \\
\hline & 0.14414 & -0.19891 & 0.04510 & -0.19779 & 0.24585 & -0.20544 \\
\hline 333.8 & 0.13941 & -0.19357 & 0.04333 & -0.19299 & 0.24013 & -0.20009 \\
\hline 334.3 & 0.13577 & -0.18937 & 0.04138 & -0.18870 & 0.23527 & -0.19603 \\
\hline 334.8 & 0.13277 & -0.18588 & 0.04039 & -0.18544 & 0.23118 & -0.19267 \\
\hline 335.8 & 0.12852 & -0.18086 & 0.03877 & -0.18055 & 0.22515 & -0.18760 \\
\hline 336.8 & 0.12299 & -0.17416 & 0.03675 & -0.17391 & 0.21692 & -0.18087 \\
\hline & 0.11785 & -0.16777 & 0.03524 & -0.16772 & 0.20895 & -0.17432 \\
\hline 338.8 & 0.11281 & -0.16139 & 0.03366 & -0.16140 & 0.20098 & -0.16763 \\
\hline
\end{tabular}

${ }^{a}$ The $x$ axis bisects the $\mathrm{Cl}-\mathrm{Cl}$ direction, and $z$ is perpendicular to the benzene ring. ${ }^{b}$ The $x$ axis lies along the $\mathrm{Cl}-\mathrm{Cl}$ direction, and $z$ is perpendicular to the benzene ring.
In order to include the layering of particles observed in smectic A phases, Kobayashi-McMillan extended MaierSaupe theory by introducing two additional terms. ${ }^{4,5}$ The first term is a sinusoidal variation of the energy as a function of the position of the particle center within a layer; this is the energy term that causes the smectic layering. The second term is a coupling between this smectic layering term and the original Maier-Saupe potential. This coupling results in a sinusoidal variation of the Maier-Saupe interaction energy as a function of position of the particle center within the layer. The liquid crystal interaction is then

$$
\begin{aligned}
H_{\mathrm{A}}^{\mathrm{lc}}= & -v_{\mathrm{lc}} S_{\mathrm{lc}} P_{2}(\cos \theta) \\
& -v_{\mathrm{lc}} \delta_{\mathrm{lc}} \alpha \tau_{\mathrm{lc}} \cos (2 \pi Z / d) \\
& -v_{\mathrm{lc}} \alpha \kappa_{\mathrm{lc}} \cos (2 \pi Z / d) P_{2}(\cos \theta) \\
= & -v_{\mathrm{lc}} S_{\mathrm{lc}} P_{2}(\cos \theta)-\tau_{\mathrm{lc}}^{\prime} \cos (2 \pi Z / d) \\
& -v_{\mathrm{lc}} S_{\mathrm{lc}} \kappa_{\mathrm{lc}}^{\prime} \cos (2 \pi Z / d) P_{2}(\cos \theta)
\end{aligned}
$$

where $S_{\mathrm{lc}}, \tau_{\mathrm{lc}}$, and $\kappa_{\mathrm{lc}}$ are the liquid crystal order parameters, $\alpha$ $=2 \exp \left[-\left(\pi r_{0} / d\right)^{2}\right], r_{0}$ is the order of the molecular length, and $d$ is the repeat distance of smectic-A translational periodicity (see ref 9, p 70, for more details). $\nu_{\mathrm{lc}}$ and $\delta_{\mathrm{lc}}$ are scale parameters characterizing interaction strengths, and $\kappa_{\mathrm{lc}}^{\prime} \equiv \alpha \kappa_{\mathrm{lc}} / S_{\mathrm{lc}}$ and $\tau_{\mathrm{lc}}^{\prime}$ $\equiv \nu_{\mathrm{lc}} \delta_{\mathrm{lc}} \alpha \tau_{\mathrm{lc}}$.

The solutes used as probes do not necessarily have axial symmetry. We restrict intermolecular interactions to those of rank two and model the anisotropic intermolecular interaction in terms of a liquid crystal dependent tensor, $F_{\gamma \delta}$, and a tensor, $\beta_{\gamma \delta}$, that depends entirely on the solute

$$
H_{\mathrm{N}}^{\mathrm{sol}}=-\frac{1}{2} F_{\gamma \delta} \beta_{\gamma \delta}
$$

where we use the Einstein convention that repeated Greek subscripts denote summation. The physical interactions that have been considered for the tensors $\mathbf{F}$ and $\boldsymbol{\beta}$ include the nematic mean square electric field interacting with the molecular polarizability, the reaction field interacting with the molecular dipole moment squared, the nematic mean electric field gradient interacting with the molecular quadrupole, and a second-rank interaction describing solute size and shape effects. ${ }^{26}$ However, the precise form of the anisotropic intermolecular interaction is not important for the current study.

The solutes used here have either $C_{2 v}$ or $D_{2 h}$ symmetry. In the uniaxial environment of the nematic phase, eq 4 can be written as

$$
H_{\mathrm{N}}^{\mathrm{sol}}=-\frac{3}{4} F_{z Z} \beta_{z z}\left(\left(\frac{3}{2} \cos ^{2}(\theta)-\frac{1}{2}\right)+\frac{b}{2} \sin ^{2}(\theta) \cos (2 \phi)\right)
$$

where

$$
b=\frac{\beta_{x x}-\beta_{y y}}{\beta_{z z}}
$$

and $Z$ is the space-fixed liquid crystal director which in the experiments reported here lies along the magnetic field; $\theta$ and $\phi$ are the polar and azimuthal angles that relate the moleculefixed $x y z$ axes to $Z$. In the simplest case, $F_{Z Z}$ is proportional to the liquid crystal mean field, $v_{\mathrm{lc}} S_{\mathrm{lc}}$. The diagonal components of the nematic-phase solute order parameter matrix calculated 
using this potential are then

$$
S_{i i}=\frac{\int \mathrm{d} \Omega\left(\frac{3}{2} \cos ^{2}\left(\theta_{i}\right)-\frac{1}{2}\right) \mathrm{e}^{-\left({ }_{\mathrm{N}}^{\mathrm{sol}} / k_{\mathrm{B}} T\right)}}{\int \mathrm{d} \Omega \mathrm{e}^{-\left({ }_{\mathrm{N}}^{\mathrm{sol}} / k_{\mathrm{B}} T\right)}}
$$

where $\theta_{i}$ is the angle between the $i$ molecular direction and $Z$.

We now assume that in the smectic A phase the solutes "see" a mean field that is similar to that experienced by the liquid crystal molecules, and in the spirit of eq 3 , we write

$$
\begin{aligned}
H_{\mathrm{A}}^{\mathrm{sol}}=-\tau^{\prime} \cos ( & 2 \pi Z / d)-\frac{3}{4} F_{Z Z} \beta_{z z}\left(\left(\frac{3}{2} \cos ^{2}(\theta)-\frac{1}{2}\right)\right. \\
+ & \left.\frac{b}{2} \sin ^{2}(\theta) \cos (2 \phi)\right)\left(1+\kappa^{\prime} \cos (2 \pi Z / d)\right)
\end{aligned}
$$

where $\tau^{\prime}$ and $\kappa^{\prime}$ are the smecticity and coupling terms. The order parameters of the solute in the smectic phase are then

$$
\begin{gathered}
S_{i i}=\int \mathrm{d} \Omega \int_{0}^{d}\left(\frac{3}{2} \cos ^{2}\left(\theta_{i}\right)-\frac{1}{2}\right) f_{\mathrm{A}}(\Omega, Z) \mathrm{d} Z \\
\tau=\int \mathrm{d} \Omega \int_{0}^{d} \cos \left(\frac{2 \pi Z}{d}\right) f_{\mathrm{A}}(\Omega, Z) \mathrm{d} Z \\
\kappa_{i i}=\int \mathrm{d} \Omega \int_{0}^{d} \cos \left(\frac{2 \pi Z}{d}\right)\left(\frac{3}{2} \cos ^{2}\left(\theta_{i}\right)-\frac{1}{2}\right) f_{\mathrm{A}}(\Omega, Z) \mathrm{d} Z
\end{gathered}
$$

where

$$
f_{\mathrm{A}}=\frac{\mathrm{e}^{-\left({ }_{\mathrm{A}}^{\mathrm{sol}} / k_{\mathrm{B}} T\right)}}{\int \mathrm{d} \Omega \int_{0}^{d} \mathrm{e}^{-\left({ }^{H_{\mathrm{A}} \mathrm{sol}} / k_{\mathrm{B}} T\right)} \mathrm{d} Z}
$$

Thus, the orientational $\left(S_{i i}\right)$ and positional $\left(\tau\right.$ and $\left.\kappa_{i i}\right)$ order parameters of each of the $C_{2 v}$ and $D_{2 h}$ solutes in the smectic A phase are described by the four parameters $\beta_{z z}, b, \tau^{\prime}$, and $\kappa^{\prime}$. Note that, in the limit $\kappa^{\prime} \rightarrow 0$ and $\tau^{\prime} \rightarrow 0$, eqs 8 and 9 revert to the nematic-phase eqs 5 and 7.

\section{Results and Discussion}

The solute NMR spectral parameters depend directly on the solute orientational order parameters, $S_{\gamma \delta}$, but do not depend directly on the smectic order parameters or the coupling between smectic and nematic order parameters. Therefore, information about the smectic terms can only be obtained indirectly from the NMR observables.

For both $8 \mathrm{CB}$ and $8 \mathrm{OCB}$, the solute and liquid crystal nematic order parameters are little affected by the nematic/smectic A phase transition. For solutes that have more than one independent $S_{\gamma \delta}$ component, the asymmetry, $R$, is essentially unaffected by the transition. However, the rate of change of this asymmetry with temperature (or any parameter such as $S_{x x}$ or $F_{z z} \beta_{x x}$ that changes with temperature) is affected (see the filled points in Figure 3), and it is this asymmetry that we use to gain information about the smectic order parameters and their coupling to the nematic order parameter.

We first fit the two independent order parameters measured in each spectrum for each of the three solutes odcb, mdcb, and pdcb to the two energy parameters $F_{Z z} \beta_{x x}$ and $b$ of the nematic potential for the solute, eq 5 (for this fitting, we rewrite eq 5 in terms of $\beta_{x x}$ instead of $\beta_{z z}$ ). The results of this fitting are presented in a plot of $b$ versus $F_{Z Z}^{\prime} \beta_{x x}$ (where $F_{Z Z}^{\prime}=F_{Z Z} / k_{\mathrm{B}}$ ) as the filled points in Figure 4. The points to the left are energies
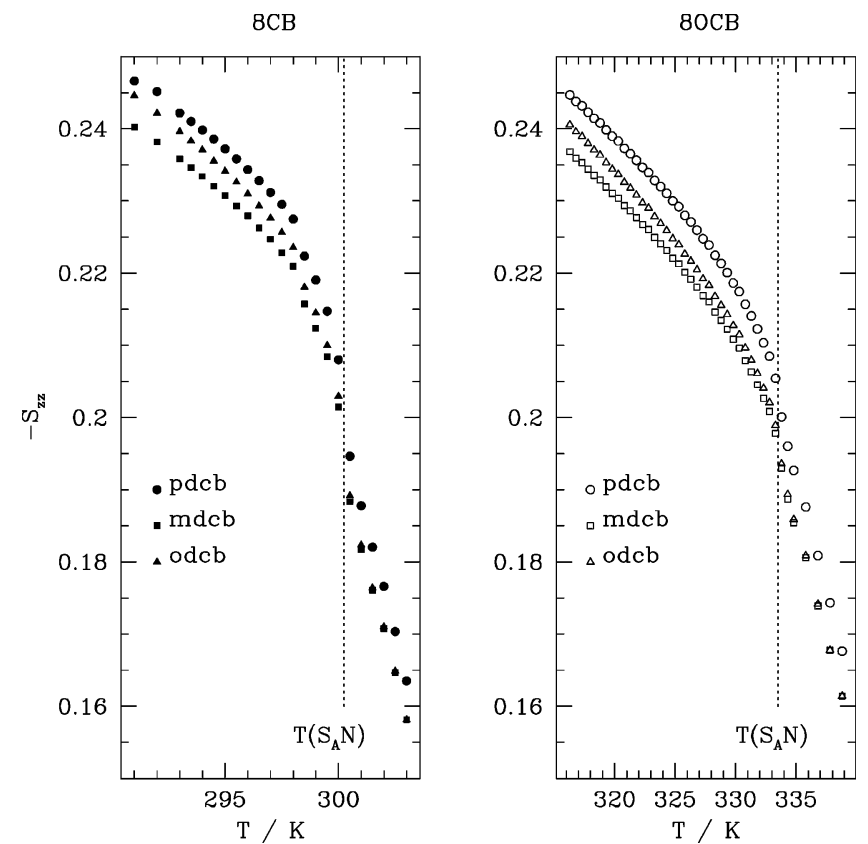

Figure 2. Variation of minus the experimental order parameter $S_{z z}$ with temperature, $T$. The smectic $\mathrm{A} /$ nematic phase transition is marked with a vertical line. The results for $8 \mathrm{CB}$ are on the left (filled symbols), and those for $80 \mathrm{OB}$ are on the right (open symbols). triangle, odcb; square, mdcb; circle, pdcb.
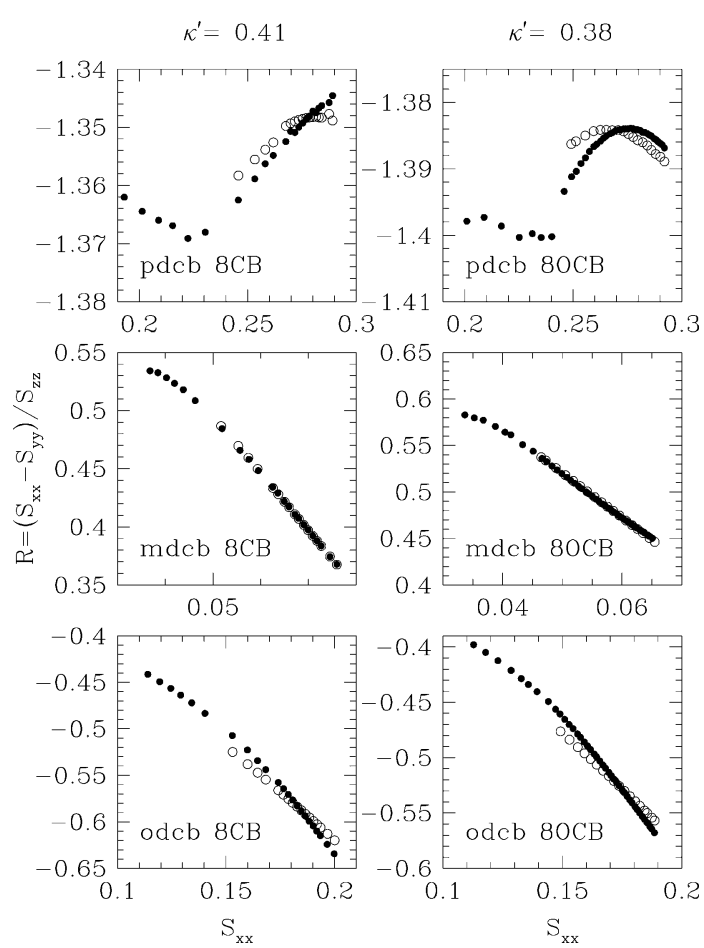

Figure 3. Order parameter ratio, $R=\left(S_{x x}-S_{y y}\right) / S_{z z}$, versus $S_{x x}$. The filled points are the experimental values, and the open circles are the values calculated from a global fit to the three solutes odcb, mdcb, and pdcb in the same liquid crystal with the same $\kappa^{\prime}$ as a variable: $\kappa^{\prime}$ $=0.41$ for $8 \mathrm{CB}$ and 0.38 for $8 \mathrm{OCB}$. The value of $\tau^{\prime \prime}$ is fitted for each solute in each liquid crystal and is scaled by $F_{Z Z} \beta_{z z}$ to give $\tau^{\prime}=\tau^{\prime \prime} F_{Z Z} \beta_{z z}$ for each experiment. The values obtained for $\tau^{\prime \prime}$ are given in Table 3 . Nematic-phase points are to the left and smectic A phase points to the right in each plot.

for the nematic phase, and those to the right are those for the smectic A phase. As was the case for the order parameter asymmetry, $R$, there is a change in slope of this plot at the 

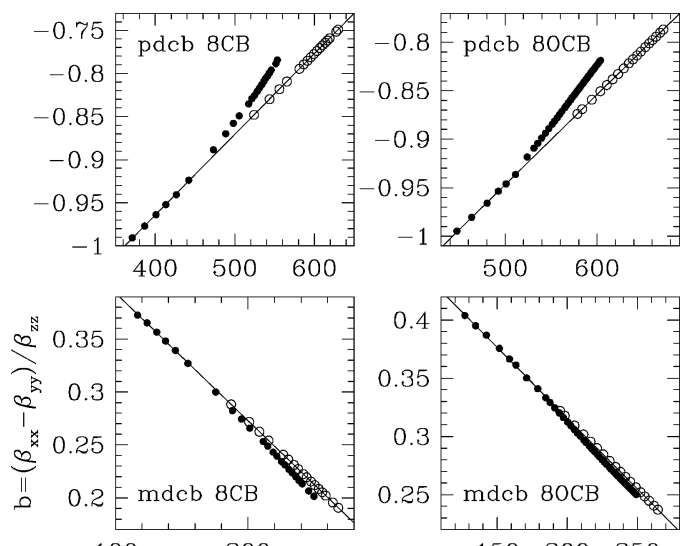

$100 \quad 200$

$150200 \quad 250$
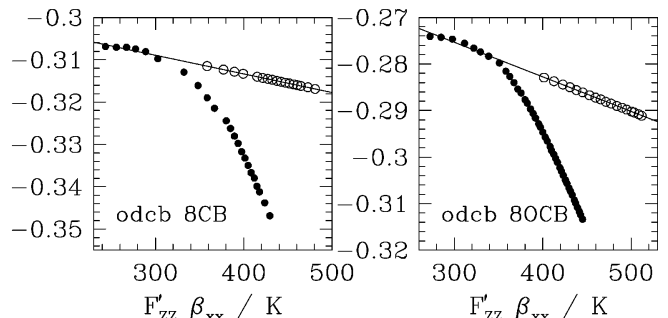

Figure 4. Asymmetry in the energy, $b$, plotted versus $F_{z z}^{\prime} \beta_{x x}$, where $F_{Z Z}^{\prime}=F_{Z Z} / k_{\mathrm{B}}$. The filled circles are the exact fitting of the solute orientational order parameters to the two independent $\beta_{i i}$ energy parameters of eqs 5 and 6 . The intercepts and slopes of the straight lines drawn through the nematic points are reported in Table 2. The open circles are the $b$ and $F_{Z Z}^{\prime} \beta_{x x}$ values from the fit to the smectic potential, eq 8 . As required by the extrapolation procedure used to obtain the nematic part of the potential in the smectic A phase, these points all fall on the nematic line. The points for the nematic phase are to the left and those for the smectic A phase to the right in each plot.

TABLE 2: Intercepts and Slopes from Fitting the Nematic $b$ Values to $b=$ Intercept + Slope $\times \boldsymbol{F}_{Z z} \boldsymbol{\beta}_{x x} / \boldsymbol{k}_{\mathrm{B}}$

\begin{tabular}{lrcccc}
\hline & \multicolumn{2}{c}{$8 \mathrm{CB}$} & & \multicolumn{2}{c}{$8 \mathrm{OCB}$} \\
\cline { 2 - 3 } \cline { 5 - 6 } solute & intercept & slope $/ \mathrm{K}^{-1}$ & & intercept & slope $/ \mathrm{K}^{-1}$ \\
\hline odcb & -0.29567 & -0.0000441 & & -0.25307 & -0.00007422 \\
mdcb & 0.51461 & -0.001209 & & 0.55903 & -0.001217 \\
pdcb & -1.33864 & 0.000936 & & -1.4114 & 0.000929
\end{tabular}

nematic/smectic A phase transition for all three solutes in both liquid crystals.

One thing to note is that $b$ is a ratio of molecular energy parameters and should be constant if there is only one interaction governing orientational order in the nematic phase and if that interaction is of tensor rank two. While fourth- and higher-rank interactions could in principle be playing a role, it seems more likely that the major contribution to the variation in $b$ involves at least two intermolecular interactions contributing to the orientational order in the nematic phases of both $8 \mathrm{CB}$ and $8 \mathrm{OCB}$

$$
H_{\mathrm{N}}^{\mathrm{sol}}=-\frac{1}{2} F_{\gamma \delta}^{(1)} \beta_{\gamma \delta}^{(1)}-\frac{1}{2} F_{\gamma \delta}^{(2)} \beta_{\gamma \delta}^{(2)} \cdots
$$

In order to explain the variation of $b$, the two (or more) interactions would have to have different temperature dependencies.

Experiments on molecular deuterium dissolved in nematic phases ${ }^{10,11,27}$ have been rationalized in terms of the orientational order of $\mathrm{D}_{2}$ being mainly due to the interaction between the solute molecular quadrupole moment and the anisotropic mean electric field gradient that $\mathrm{D}_{2}$ sees in the nematic environment. Experiments on larger solutes have been rationalized in terms of short-range interactions that are governed by the solute size and shape. ${ }^{10,11}$ It is possible that these two interactions are
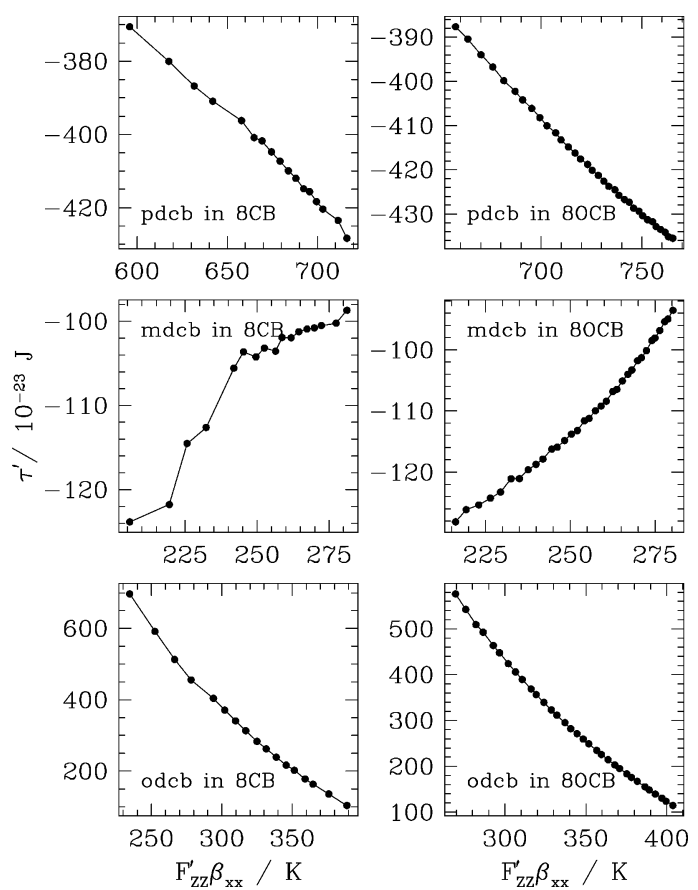

Figure 5. Fitting parameter $\tau^{\prime}$ versus $F_{z z}^{\prime} \beta_{x x}=F_{Z z} \beta_{x x} / k_{\mathrm{B}}$ for exact fits to each experiment. The value of $b$ was calculated from $F_{Z Z}^{\prime} \beta_{x x}$ using linear extrapolation from the nematic-phase values; $\kappa^{\prime}$ was fixed to 0.65 . Note that similar exact fits are possible with other values of $\kappa^{\prime}$.

operative in the liquid crystals of this study and that their different temperature dependencies cause the temperature dependence of $b$.

Here, we are interested in the smectic order, and we shall not worry further about the specifics of the interactions that lead to the nematic ordering. However, in order to proceed, we must make some assumptions and approximations. In most cases, the nematic-phase points in Figure 4 fall on a reasonably straight line. Each experiment yields two independent order parameters, and the filled points of Figure 4 are the two independent energy parameters $F_{Z z}^{\prime} \beta_{x x}$ and $b$ (related to eq 5 for the nematic phase) that reproduce the experimental order parameters using eq 7 . There appear to be no parameters remaining to allow a fit to the smectic parameters $\tau^{\prime}$ and $\kappa^{\prime}$. In order to proceed, we need to reduce the number of variables required to fit the nematic part of the potential in the smectic A phase.

Note in Figure 4 that the smectic points for each solute in $8 \mathrm{CB}$ and $80 \mathrm{CB}$ show deviations from the linear behavior of the nematic points. We now assume that the variation of the nematic-phase points for each solute and each liquid crystal in Figure 4 represents how the nematic part of the potential would vary in the smectic A phase-in other words, we assume that $b$ and $F_{Z z} \beta_{x x}$ are correlated in the same way in all phases. Hence, we fit a linear regression straight line to the nematic points of Figure 4 (values given in Table 2) and use the slopes and intercepts to describe the correlations. Effectively, this frees up one of the two experimentally determined $S$ parameters for each experiment at low temperatures, and we can therefore attempt to use this free parameter to fit the smectic and coupling parameters to the experimental results.

To proceed, we extrapolate the nematic points of Figure 4 into the smectic A phase. In principle, we can now adjust one extra parameter for each experiment in the smectic A phase. Unfortunately, we see in eq 8 that we need to determine both a $\tau^{\prime}$ value and a $\kappa^{\prime}$ value for each solute in each experiment. We are forced to make further assumptions. 
TABLE 3: Fitted Smectic A Parameters ${ }^{a}$

\begin{tabular}{|c|c|c|c|c|c|c|}
\hline calculation & & $\kappa^{\prime}$ & $\tau^{\prime \prime b}$ & $\kappa_{x x}$ & $\kappa_{z z}$ & $\tau$ \\
\hline $8 \mathrm{CB}$ & odcb & $0.408(11)$ & $0.232(39)$ & -0.001 to 0.006 & -0.002 to 0.012 & -0.182 to -0.148 \\
\hline global fit & mdcb & $0.408(11)$ & $0.120(4)$ & $0.002-0.002$ & -0.022 to -0.022 & -0.049 to -0.047 \\
\hline vary $\kappa^{\prime}$ & pdcb & $0.408(11)$ & $0.319(6)$ & -0.018 to -0.001 & $0.011-0.029$ & -0.252 to -0.208 \\
\hline $8 \mathrm{CB}$ & odcb & 0.65 fixed & -1.41 to -0.13 & $0.095-0.114$ & -0.149 to -0.108 & $0.244-0.660$ \\
\hline exact fit & mdcb & 0.65 fixed & $0.10-0.16$ & $0.005-0.013$ & -0.061 to -0.043 & -0.049 to 0.021 \\
\hline fix $\kappa^{\prime}=0.65$ & pdcb & 0.65 fixed & $0.36-0.40$ & $0.019-0.020$ & $0.000-0.007$ & -0.261 to -0.256 \\
\hline $8 \mathrm{CB}$ & odcb & 0.50 fixed & -1.11 to 0.22 & $0.017-0.106$ & -0.139 to -0.008 & -0.140 to 0.606 \\
\hline exact fit & mdcb & 0.50 fixed & $0.11-0.14$ & $0.002-0.007$ & -0.036 to 0.022 & -0.257 to -0.024 \\
\hline fix $\kappa^{\prime}=0.50$ & pdcb & 0.50 fixed & $0.33-0.34$ & -0.006 to 0.009 & -0.052 to 0.014 & -0.240 to 0.047 \\
\hline $8 \mathrm{OCB}$ & odcb & $0.377(9)$ & $0.310(34)$ & -0.024 to -0.009 & $0.018-0.039$ & -0.280 to -0.226 \\
\hline global fit & mdcb & $0.377(9)$ & $0.103(3)$ & $0.001-0.001$ & -0.023 to -0.022 & -0.037 to -0.036 \\
\hline vary $\kappa^{\prime}$ & pdcb & $0.377(9)$ & $0.298(4)$ & -0.015 to -0.001 & $0.010-0.025$ & -0.229 to -0.191 \\
\hline $80 C B$ & odcb & 0.65 fixed & -0.99 to -0.13 & $0.089-0.102$ & -0.137 to -0.106 & $0.240-0.562$ \\
\hline exact fit & mdcb & 0.65 fixed & $0.09-0.15$ & $0.004-0.010$ & -0.063 to -0.044 & -0.040 to 0.031 \\
\hline fix $\kappa^{\prime}=0.65$ & pdcb & 0.65 fixed & $0.35-0.38$ & $0.025-0.026$ & -0.005 to 0.000 & -0.242 to -0.236 \\
\hline $8 \mathrm{OCB}$ & odcb & 0.50 fixed & -0.76 to 0.13 & $0.034-0.092$ & -0.123 to -0.038 & -0.014 to 0.498 \\
\hline exact fit & mdcb & 0.50 fixed & $0.09-0.13$ & $0.002-0.006$ & -0.036 to 0.014 & -0.230 to -0.022 \\
\hline fix $\kappa^{\prime}=0.50$ & pdcb & 0.50 fixed & $0.32-0.33$ & $0.004-0.013$ & $0.001-0.008$ & -0.220 to -0.200 \\
\hline
\end{tabular}

${ }^{a}$ Errors in the least significant digits are in parentheses. The ranges given are for all experiments performed in the smectic A phase. ${ }^{b} \tau^{\prime \prime}=$ $\tau^{\prime} / F_{z z} \beta_{z z}$. Note that $\beta_{z z}$ is negative and therefore $\tau^{\prime}$ and $\tau^{\prime \prime}$ have opposite signs.

If all solutes (and possibly the liquid crystal molecules) experience the same orientational mechanism, then there are excellent physical grounds for assuming that the coupling term, $\kappa^{\prime}$, is a liquid crystal property and is therefore the same for all solutes, and in fact is related to that for the liquid crystal itself. As noted above, the variation of $b$ in the nematic phase indicates that more than one anisotropic intermolecular interaction is causing the orientational order and therefore fixing $\kappa^{\prime}$ to the same value for all solutes is not rigorously justifiable. However, this coupling term essentially describes how the nematic potential varies as we move across a smectic layer and it is reasonable to assume that all contributions to this nematic potential experience the same variation, and hence, we shall require that $\kappa^{\prime}$ be a liquid crystal property and be the same for all solutes in a particular liquid crystal.

Both $\tau^{\prime}$ and $\kappa^{\prime}$ are expected to vary with temperature. We see that $\kappa^{\prime}$ is multiplied by $F_{Z Z} \times \beta$ parameters in the smectic A Hamiltonian (eq 8). We shall assume that the temperature dependence of $\kappa^{\prime}$ is accounted for by the temperature dependence of $F_{Z Z} \beta$; consequently, we treat $\kappa^{\prime}$ as an adjustable constant parameter of the liquid crystal.

If we wish to fit each experiment separately, as stated above, we can fit only one smectic parameter $\left(\kappa^{\prime}\right.$ or $\left.\tau^{\prime}\right)$ per experiment, and hence, we must choose a value for one of these. Preliminary calculations show that the values of $\kappa^{\prime}$ and $\tau^{\prime}$ are somewhat correlated. As a first approach to fitting the results, we fix $\kappa^{\prime}$ to a "reasonable" value and fit the remaining two parameters, $F_{Z z} \beta_{x x}$ and $\tau^{\prime}$, for each solute in each experiment.

We can achieve exact fits to all experiments for values of $\kappa^{\prime}$ $\geq 0.5$, and to most experiments for lower values of $\kappa^{\prime}$. We show the exact fits for $\kappa^{\prime}=0.65$ in Figure 5 where we plot the fitted values of $\tau^{\prime}$ versus $F_{Z z}^{\prime} \beta_{x x}$. Intuitively, we might expect absolute values of $\tau^{\prime}$ to increase with the nematic potential term $F_{Z z} \beta_{x x}$; that is, the points in Figure 5 should fit lines of negative slope, as is the case for the pdcb results. $\tau^{\prime}$ does not vary much for mdcb, but the variation is counter to the above intuition. The odcb results do have a negative slope, but the absolute value of $\tau^{\prime}$ (which is positive) decreases. The exact fits obtained for $\kappa^{\prime}=0.50$ (not shown) have the same general slope, and for both pdcb and mdcb, the values of $\tau^{\prime}$ and $F_{Z Z} \beta_{x x}$ are slightly smaller. For odcb, the values of $F_{Z z} \beta_{x x}$ are larger and $\tau^{\prime}$ becomes negative at lower temperatures.

Because of the simplifications we have made, not too much significance should be placed in the numbers obtained from these exact fits. The ranges of the smectic order parameters obtained are given in Table 3.

The problem with the exact fits above is the criteria to use to choose an appropriate value for $\kappa^{\prime}$. Examination of eq 8 shows that the value $\kappa^{\prime}=1$ would have the nematic potential $H_{\mathrm{N}}^{\mathrm{sol}}=$ 0 at $Z=d / 2$, hence giving an isotropic region within the smectic layer. Such isotropic regions are unlikely, even in the hydrocarbon region of the smectic layers. A reasonable upper limit on the variation of the nematic potential as a function of position in the smectic layers might well be that given by $\kappa^{\prime}=0.65$, with smaller values being quite acceptable.

As a second approach to fitting the results, and in order to get around the necessity of choosing a value for $\kappa^{\prime}$, we shall assume a temperature dependence for $\tau^{\prime}$ and perform a fit to all experiments for all solutes in a given liquid crystal: the fitting parameters include one $\kappa^{\prime}$ value for each liquid crystal, parameter(s) to describe the temperature dependence of $\tau^{\prime}$ for each solute in each liquid crystal, as well as $F_{z z} \beta_{x x}$ values for each solute at each temperature. We note in eq 3 for KobayashiMcMillan theory applied to the liquid crystal that the temperature dependence of both the nematic and coupling terms is achieved by the scaling factor $S_{\mathrm{lc}}$ which is temperature dependent. In eq 8 for solutes, both nematic and coupling terms contain the factor $F_{z Z}$ multiplied by $\beta_{x x}, \beta_{y y}$, and $\beta_{z z}$ and by angle factors; $F_{Z Z}$, the nematic mean potential, is temperature dependent and serves the same scaling function for the solute as does $S_{\mathrm{lc}}$ for the solvent. In the absence of arguments to the contrary, it seems quite reasonable to assume that the smectic term, $\tau^{\prime}$, in eq 8 for solute ordering in smectic A phases has a temperature dependence similar to the nematic and coupling terms. Figure 2 shows that for the solutes considered here $S_{z z}$ (the order parameter for the direction perpendicular to the ring) takes roughly the same value for all solutes in any given experiment. Hence, $F_{Z z} \beta_{z z}$ would seem to be the sensible choice for scaling $\tau^{\prime}$. We note that the linear temperature dependence of $\tau^{\prime}$ reported in ref 6 is not used here partly because the resulting $\kappa^{\prime}$ values are close to 1 . Hence, we set $\tau^{\prime}=\tau^{\prime \prime} F_{Z z} \beta_{z z}$, where $\tau^{\prime \prime}$ is a fitting parameter for each solute in each liquid crystal.

The adjustable parameters are then a single value of $\kappa^{\prime}$ for the liquid crystal, three values of $\tau^{\prime \prime}$ (one for each solute), and a value of $F_{Z Z} \beta_{x x}$ for each solute at each temperature. The value 

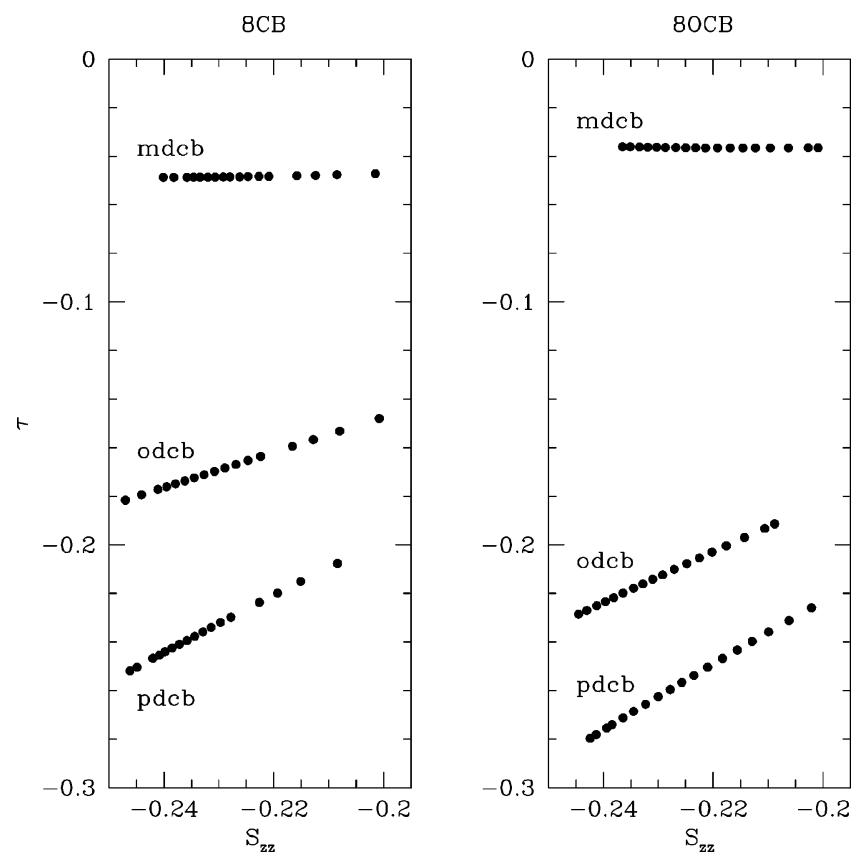

Figure 6. Smectic order parameters, $\tau$, versus $S_{z z}$ obtained from the global fit of odcb, mdcb, and pdcb orientational order parameters in the liquid crystals $8 \mathrm{CB}$ and $8 \mathrm{OCB}$.

of $F_{Z Z} \beta_{z z}$ used for scaling is calculated using eq 6 and the value of $b$ obtained from $F_{Z z} \beta_{x x}$ and the slope and intercept in Table 2 .

The open points in Figure 4 give the values of $b$ and $F_{z z} \beta_{x x} /$ $k_{\mathrm{B}}$ obtained from this fitting. As required by the fitting procedure, the calculated values all lie on the line extrapolated from the nematic-phase points. The fitted values of $\kappa^{\prime}$ are 0.41 for $8 \mathrm{CB}$ and 0.38 for $8 \mathrm{OCB}$. Other parameters and the smectic order parameters obtained from the fitting are given in Table 3.

The nematic order parameters calculated from eq 9 for this fit (open circles) are compared with the experimental values (filled circles) in Figure 3. While the fit is not perfect, it does follow the general trends observed experimentally. For pdcb, a much improved fit results for $\kappa^{\prime} \approx 0.5$; however, then, the fit for odcb is poor. Larger errors for odcb could also arise from the fact that $b$ in the nematic phase for odcb is in fact markedly nonlinear (thus, a linear fit such as the one shown in Figure 4 might not be appropriate in this case). Considering the approximations made and the assumption that all energy parameters scale with $F_{Z Z}$, the agreement between fitted and experimental values in Figure 3 is excellent, and demonstrates that Kobayashi-McMillan theory is useful for describing solutes in smectic A liquid crystals.

We also use the fitted parameters to calculate the smectic and coupling order parameters from eqs 10 and 11 . The calculated smectic order parameters, $\tau$, for the fit in which $\kappa^{\prime}$ is adjusted are displayed in Figure 6. The $\tau$ values differ among solutes but are quite similar for a given solute in both liquid crystals. Except for mdcb (where $\tau$ is small), values are slightly larger in magnitude in $8 \mathrm{OCB}$ compared to $8 \mathrm{CB}$. The liquid crystal structures differ by one oxygen, and this difference has little effect on the smectic ordering of the solutes studied here; far larger differences are noted among the solutes themselves. The $\tau$ values decrease as $S_{z z}$ decreases, indicating as expected that both the nematic and smectic potentials increase in magnitude at lower temperatures.

In both liquid crystals, the $\tau$ values for mdcb are very small, indicating that mdcb is spread relatively evenly throughout the smectic layers. The $\tau$ values for pdcb are of the order -0.25 , indicating that this solute tends to partition more than mdcb does in selected regions of the layers. The behavior of odcb lies between that for mdcb and pdcb.

The relative signs of $\tau^{\prime}$ and $\kappa^{\prime}$ are determined by the fitting; unfortunately, the absolute signs are unknown and it is not possible to say with certainty which region odcb and pdcb prefer. However, we anticipate (indeed have assumed) that the coupling term, $\kappa^{\prime}$, is closely related to $\kappa_{\mathrm{lc}}$. As the liquid crystal has a "rigid" core and "floppy" hydrocarbon tails, we expect the orientational potential to be minimum (maximum orientational order) near the core (center of a layer) and maximum (minimum orientational ordering) at the interface between layers. These ideas are accommodated when we place the $Z$ origin at the center of a layer and set $\kappa^{\prime}$ positive in our calculations.

We note that $\tau^{\prime \prime}$ for the global fits (in Table 3 ) and $\beta_{z z}$ are of opposite sign, meaning that $\tau^{\prime}$ is negative in all cases. Hence, eq 8 then predicts that the smectic potential is maximum at the origin and minimum at the interlayer interface, indicating that the solutes prefer to partition near the interface (hydrocarbon tail region of the liquid crystal molecules), with the partitioning being stronger for odcb and pdcb than for mdcb. Preference for the interlayer region agrees with earlier observations. ${ }^{16}$

Ranges of values of the smectic order parameters, $\tau$, calculated from eq 10 for other fits, including the exact fits with assumed values of $\kappa^{\prime}$, are given in Table 3 . The values for mdcb and pdcb are relatively insensitive to the various assumptions made in the various calculations. The values for odcb do depend on the calculation and change sign from negative (in the global fit with $\kappa^{\prime} \approx 0.4$ ) to positive for larger vales of $\kappa^{\prime}$. This change of sign is associated with the change of sign of $\tau^{\prime}$ found for fits with the larger $\kappa^{\prime}$ values. However, the smaller values associated with the global fits with $\kappa^{\prime}$ varied are probably reasonable.

Ranges for the coupling order parameters, $\kappa_{i i}$, are also given in Table 3. As seen by comparing eqs 9 and 11, the $\kappa_{i i}$ values are essentially scaled by $S_{i i}$. As $S_{z z}$ is essentially the same for all solutes in a given experiment, it seems sensible to compare $\kappa_{z z}$ values. The general observation is that all $\kappa_{z z}$ values are small, especially for the global fits. The values do change a lot with different assumed values for $\kappa^{\prime}$, and therefore, it is difficult to say more about them.

\section{Conclusions}

In this paper, we have presented a way of probing the anisotropic intermolecular potential in uniaxial nematic and smectic A phases. We have extended our investigation of solutes in liquid crystals that form both nematic and lower-temperature smectic A phases to the investigation of two additional solutes in two liquid crystals. We have used a larger range of $F_{z z} \beta_{x x}$ and $b$ values in the new work in order to more critically test the theory. The original ideas that were based on the analysis of only two solutes in the single liquid crystal $8 \mathrm{CB}$ still hold. Smaller values of $\kappa^{\prime}$ are required to fit the results in this compared to the earlier work, and this is due to the linear scaling with temperature (as opposed to scaling with the nematic potential) used for $\tau^{\prime}$ in the previous study. ${ }^{6}$

We have shown that Kobayashi-McMillan theory, which was developed as a model for orientational and positional ordering in smectic A phases, can rationalize the NMR results for solutes in these phases. The energy asymmetry values, $b$, for odcb (Figure 4) are curved even in the nematic phase. Even so, we are able to fit the results to a straight line in the same way as we do the results for the other two solutes, mdcb and pdcb. It may be that failure to account fully for the curvature leads to 
the strong dependence we observe in odcb of $\tau^{\prime}$ on $\kappa^{\prime}$, and also to the fact that the recalculated order parameter asymmetries in Figure 3 fit less well for the solute odcb. The pdcb results in Figure 3 also do not appear to fit well; however, they do mimic the curvature found experimentally for $80 \mathrm{CB}$, and the fit for pdcb is much improved if we increase the value of $\kappa^{\prime}$ to 0.50 . The problem with the odcb results is that the experimental and calculated slopes in Figure 3 are different, and this difference does not change significantly with different values of $\kappa^{\prime}$; the difference in slope for odcb is a function of the assumed scaling of $\tau^{\prime}$ by $\beta_{z z}$ as well as the assumed linear dependence of $b$ in the nematic phase.

The relative values of $\tau$ obtained from the analysis indicate that mdcb is evenly distributed throughout the smectic layers while pdcb appears to be somewhat localized at one position within the layers. On the basis of the different signs we obtain for $\kappa^{\prime}$ and $\tau^{\prime}$, this position is likely the interlayer (i.e., hydrocarbon tail) region of the layers, in agreement with earlier observations in ref 16 . The odcb behaves in between mdcb and pdcb.

There remain problems. For example, $\tau^{\prime}$ and $\kappa^{\prime}$ are somewhat correlated, and in the current analysis, we are forced to make assumptions and approximations in order to fit the results obtained from the NMR experiments. Nevertheless, we have demonstrated that the simple Kobayashi-McMillan ordering mechanism for smectic phases works well for solutes in smectic A phases, and we are able to determine the parameters of the theory as well as the solute nematic, smectic, and coupling order parameters. The relative signs determined for $\kappa^{\prime}$ and $\tau^{\prime}$ in combination with the magnitude of the smectic order parameter, $\tau$, give information about the partitioning of the solutes within the smectic layers.

Acknowledgment. We acknowledge financial support from the Natural Sciences and Engineering Research Council of Canada.

\section{References and Notes}

(1) Gray, G. W., Vill, V., Spiess, H. W., Demus, D., Goodby, J. W., Eds. Physical Properties of Liquid Crystals; Wiley-VCH: Weinheim, NY, 1999.

(2) Gaemers, S.; Bax, A. J. Am. Chem. Soc. 2001, 123, 12343.

(3) Burnell, E. E.; de Lange, C. A. Chem. Phys. Lett. 1980, 76, 268

(4) Kobayashi, K. K. Mol. Cryst. Liq. Cryst. 1971, 13, 137.

(5) McMillan, W. L. Phys. Rev. A 1971, 4, 1238.

(6) Yethiraj, A.; Sun, Z.; Dong, R. Y.; Burnell, E. E. Chem. Phys. Lett. 2004, 398, 517. A typographical sign error in the definition of $P_{2 \text {,asymm }}$ following eq 4 is corrected in eq 5 of the present paper; $b$ in Figure 2 should be replaced by $-b$.

(7) Als-Nielsen, J.; Litster, J. D.; Birgeneau, R. J.; Kaplan, M.; Safinya, C. R.; Lindegaard-Andersen, A.; Mathiesen, S. Phys. Rev. B 1980, 22, 312.

(8) Link, S.; Chang, W-S.; Yethiraj, A.; Barbara, P. F. Phys. Rev. Lett. 2006, 96, 17801.

(9) Dong, R. Y. Nuclear Magnetic Resonance of Liquid Crystals, 2nd ed.; Springer-Verlag: New York, 1994.

(10) Burnell, E. E.; de Lange, C. A. Prediction from molecular shape of solute orientational order in liquid crystals. Chem. Rev. 1998, 98, 2359.

(11) Burnell, E. E., de Lange, C. A., Eds. NMR of Ordered Liquids; Kluwer Academic: Dordrecht, The Netherlands, 2003.

(12) Luz, Z.; Meiboom, S. J. Chem. Phys 1973, 59, 275.

(13) Catalano, D.; Marcolini, L.; Veracini, C. A. Chem. Phys. Lett. 1982 88,342 .

(14) Vaz, N. A. P.; Doane, J. W. J. Chem. Phys 1983, 79, 2470.

(15) Catalano, D.; Forte, C.; Veracini, C. A.; Emsley, J. W.; Shilstone, G. N. Liq. Cryst. 1987, 2, 357.

(16) Cinacchi, G. Chem. Phys. Lett. 2005, 416, 238.

(17) Goldfarb, D.; Luz, Z.; Zimmermann, H. J. Phys. 1982, 43, 421.

(18) Goldfarb, D.; Luz, Z.; Zimmermann, H. J. Phys. 1982, 43, 1255

(19) Huang, Z.; Sandstrom, U.; Henriksson, A.; Maliniak, J. J. Phys. Chem. B 1998, 102, 8395.

(20) Maier, W.; Saupe, A. Z. Naturforsch., A 1959, 14, 882.

(21) Maier, W.; Saupe, A. Z. Naturforsch., A 1960, 15, 287.

(22) Diehl, P.; Kellerhals, H.; Niederberger, W. J. Magn. Reson. 1971, 4,352 .

(23) Syvitski, R. T.; Burnell, E. E. Can. J. Chem. 1999, 77, 1761.

(24) Syvitski, R. T.; Burnell, E. E. J. Magn. Reson. 2000, 144, 58.

(25) Diehl, P.; Henrichs, P. M.; Niederberger, W. Mol. Phys. 1971, 20, 139.

(26) Syvitski, R. T.; Burnell, E. E. J. Chem. Phys. 2000, 113, 3452.

(27) ter Beek, L. C.; Burnell, E. E. Chem. Phys. Lett. 2006, 426, 96. 